\title{
Pattern-based sensing of nucleotides in aqueous solution with a multicomponent indicator displacement assay $\dagger$
}

\author{
Andrey Buryak, ${ }^{a}$ Alexei Pozdnoukhov ${ }^{b}$ and Kay Severin*a \\ Received (in Cambridge, UK) 10th April 2007, Accepted 4th May 2007 \\ First published as an Advance Article on the web 17th May 2007 \\ DOI: 10.1039/b705250b
}

\begin{abstract}
A multicomponent indicator displacement assay (MIDA) based on an organometallic receptor and three dyes can be used for the identification and quantification of nucleotides in aqueous solution at neutral $\mathrm{pH}$.
\end{abstract}

Nucleotides are of prime importance for many biological processes. It is therefore not surprising that considerable efforts have been made to build artificial receptors and sensors for this class of compounds. ${ }^{1}$ Despite significant success, many of the systems described so far display limitations such as low sensitivity or selectivity, or require substantial synthetic efforts. In the following we describe an indicator displacement assay (IDA), which is based on commercially available building blocks. In combination with statistical tools such as linear discriminant analyses or artificial neural networks, it is possible to sense nucleotides in aqueous solution by UV-Vis spectroscopy with high fidelity.

A synthetic receptor, which is bound via non-covalent interactions to an indicator, can function as a sensor, given that the displacement of the indicator by an analyte results in a change of its optical properties. ${ }^{2}$ Generally, a particular indicator is chosen so that its affinity for the receptor is lower than that of the analyte of interest but higher than that of competing analytes. ${ }^{2,3}$ The analysis is then performed at the wavelength, where the free and the bound indicator displays the largest difference in absorption (colorimetric sensor) or emission (fluorescent sensor). Although the majority of IDAs have been performed according to this scheme, there are first reports, which suggest that the parallel utilization of multiple receptors and/or indicators in a single cuvette can be advantageous. Anslyn et al. have used a MIDA based on two receptors and two indicators to simultaneously sense malate and tartrate. ${ }^{4}$ Subsequently, the same group has shown that citrate and calcium can be determined with an IDA, in which one analyte acts as a second receptor. ${ }^{5}$ We have recently demonstrated that a MIDA based on a dynamic mixture of several metal-dye complexes can be used to sense di- and tripeptides. ${ }^{6}$

A direct consequence of using multiple receptors/indicators is that the dynamic range of the assay can be enlarged. For a classical one-receptor-one-indicator IDA, analytes which show a significantly higher or lower affinity to the receptor than the indicator

${ }^{a}$ Institut des Sciences et Ingénierie Chimiques, École Polytechnique Fédérale de Lausanne (EPFL), 1015, Lausanne, Switzerland.

E-mail: kay.severin@epfl.ch; Fax: +41(0)216939305;

Tel: +41(0)216939302

${ }^{b}$ Institute of Geomatics and Analysis of Risk, University of Lausanne

(UNIL), 1015, Lausanne, Switzerland

$\uparrow$ Electronic supplementary information (ESI) available: Experimental details. See DOI: 10.1039/b705250b give rise to the same all or nothing signal. In MIDAs, low-affinity analytes and high-affinity analytes may give rise to a signal. For a sensor based on one receptor and three different indicators, this is shown in Scheme 1. The assembly of the receptor with the dyes leads to the formation of three sensors, which give a differential response upon addition of analytes. One should note that the changes for the various indicator/receptor combinations need to occur in different regions of the spectrum in order to provide a good differentiation. As a consequence, the analysis is performed with the data of several wavelengths and pattern recognition protocols are valuable tools to assist the analysis. The MIDA approach is conceptually related to sensor arrays. ${ }^{7}$ But contrary to sensor arrays with independent sensor units, the sensors used in a MIDA are connected by exchange reactions. Furthermore, the various sensors of an array are generally addressed separately whereas for a MIDA, a single measurement is sufficient as the read-out.

Previously, we have shown that the organometallic complex $\left[\mathrm{Cp}^{*} \mathrm{RhCl}_{2}\right]_{2}$ (1) (Fig. 1) can be used to construct IDAs for the sensing of His/Met containing peptides, ${ }^{8}$ amino acids, ${ }^{9}$ and aminosugars. ${ }^{10}$ It had been reported that the $\mathrm{Cp} * \mathrm{Rh}$ fragment is able to bind to nucleotides in aqueous solution. ${ }^{11}$ This prompted us to investigate, whether $\mathbf{1}$ can be used to build a MIDA for nucleotides. Suited indicators for a MIDA should fulfill the following criteria: (a) they should bind to the $\mathrm{Cp} * \mathrm{Rh}$ fragment with different affinities; (b) the spectroscopic changes upon coordination should occur in different regions of the UV-Vis spectrum. From a small screening of commercially available dyes, the following three indicators emerged as potential candidates: Mordant Yellow 10 (2), Gallocyanine (3) and Evans Blue (4). In buffered aqueous solution (50 mM phosphate buffer, $\mathrm{pH} 7.4$ ), Mordant Yellow 10 binds to the $\mathrm{Cp} * \mathrm{Rh}$ fragment with an affinity of $K_{\mathrm{a}}=3.7( \pm 0.3) \times 10^{4} \mathrm{M}^{-1}$ (see ESI $\left.\dagger\right)$ and major changes upon

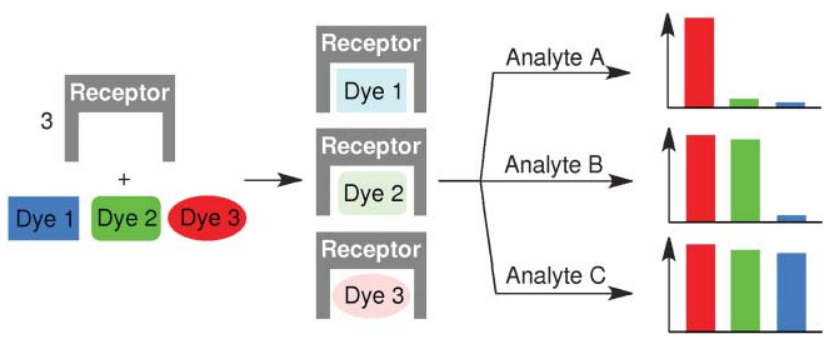

Scheme 1 Schematic representation of a MIDA based on one receptor and three different indicators. Analytes with a low affinity for the receptor lead to a displacement of weakly bound indicators whereas high-affinity analytes displace all indicators. 
<smiles></smiles>

1<smiles>CN(C)c1ccc2nc3c(C(=O)O)cc(=O)c(O)c-3oc2c1</smiles>

2

Mordant Yellow 10

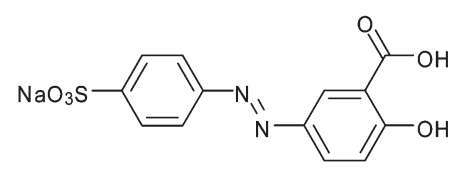

Fig. 1 The Rh complex and the three dyes, which were used to build the sensor.

binding occur at $408 \mathrm{~nm}$. Gallocyanine shows an affinity of $K_{\mathrm{a}}=$ $2.3( \pm 0.2) \times 10^{6} \mathrm{M}^{-1}$ and spectral changes occur at 625 and $720 \mathrm{~nm} .{ }^{9}$ The affinity of Evans Blue to the Rh complex was found to be higher than that of Gallocyanine but due to the presence of multiple metal binding sites, an association constant was not calculated. The most significant spectral changes occur at $601 \mathrm{~nm}$. A comparison of the spectral properties of the three dyes is shown in Fig. 2.

Next, we investigated the response of a sensor comprised of complex $1([\mathrm{Rh}]=120 \mu \mathrm{M})$ and the dyes 2, 3 and $\mathbf{4}([2]=[3]=$ $40 \mu \mathrm{M},[4]=20 \mu \mathrm{M})$ upon addition of the nucleotides adenosine diphosphate (ADP), adenosine triphosphate (ATP) or guanosine triphosphate $(\mathrm{GTP})([$ nucleotide $]=0.5 \mathrm{mM})$. For ADP, the most pronounced changes occurred at $443 \mathrm{~nm}$ indicating that only the weakly bound Mordant Yellow 10 was displaced to a significant extend (Fig. 3). For GTP, changes at $619 \mathrm{~nm}$ were observed in addition to changes at $436 \mathrm{~nm}$. This showed that GTP has a higher affinity for the $\mathrm{Rh}$ complex than $\mathrm{ADP}$, resulting in a replacement of Mordant Yellow 10 and Gallocyanine. For ATP, the maximum changes in the UV-Vis spectrum occurred around $620 \mathrm{~nm}$, which

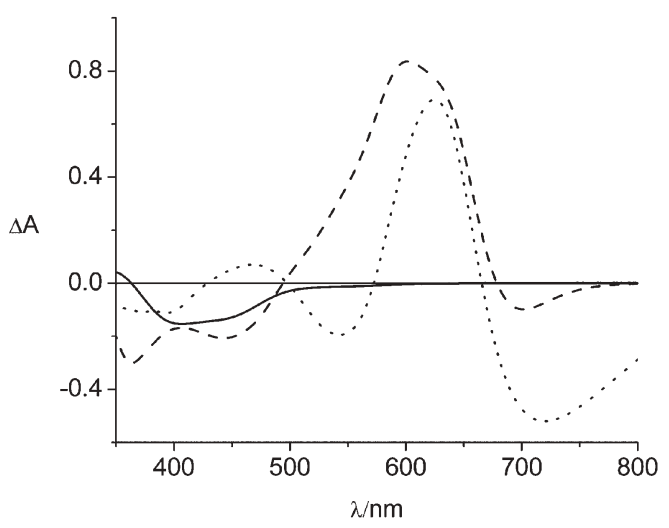

Fig. 2 UV-Vis difference spectra obtained upon coordination of Mordant Yellow 10 (solid line), Gallocyanine (dotted line) or Evans Blue (dashed line) to 1. Concentrations: [Mordant Yellow 10] $=40 \mu \mathrm{M}$, $[$ Gallocyanine $]=40 \mu \mathrm{M}$, [Evans Blue $]=20 \mu \mathrm{M},[\mathrm{Rh}]=40 \mu \mathrm{M} ; 50 \mathrm{mM}$ phosphate buffer, $\mathrm{pH} 7.4$.

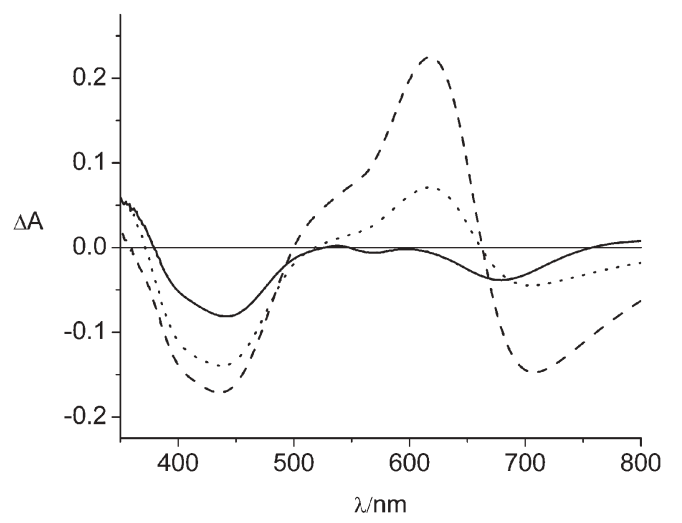

Fig. 3 UV-Vis difference spectra obtained upon addition of ADP (solid line), GTP (dotted line) or ATP (dashed line) to a sensing ensemble composed of complex $\mathbf{1}$ and the dyes 2-4. Concentrations: [Mordant Yellow 10] $=[$ Gallocyanine $]=40 \mu \mathrm{M}$, [Evans Blue $]=20 \mu \mathrm{M},[\mathrm{Rh}]=$ $120 \mu \mathrm{M}$, [nucleotide] $=0.5 \mathrm{mM}, 50 \mathrm{mM}$ phosphate buffer, $\mathrm{pH} 7.4$.

suggested that this analyte is also able to at least partially displace the high affinity dye Evans Blue.\$ A MIDA based on complex 1 and the dyes 2-4 therefore allows an easy distinction of these three nucleotides.

To test the scope of our sensor, we first extended the range of the analytes by adding cyclic adenosine monophosphate (cAMP), adenosine monophosphate (AMP) and the pyrophosphate anion (PPi). We then performed a similar experiment but this time we employed a linear discriminant analysis (LDA) ${ }^{12}$ to identify the analytes. $\S$ Six independent measurements were performed for each analyte $([$ nucleotide $]=[\mathrm{PPi}]=1.0 \mathrm{mM})$. The intensities at the wavelengths $720,625,600,540$ and $440 \mathrm{~nm}$ were chosen as the input variables for the LDA. The final score plot is shown in Fig. 4. The data appear in well-separated groups, which demonstrates that the six analytes can readily be discriminated.

The very good differentiation that was achieved prompted us to attempt a more difficult task: to determine simultaneously the concentrations of several analytes. For this purpose, we have focused on ATP, cAMP, and PPi. The hydrolysis of ATP to give cAMP and PPi is catalyzed by adenylate cyclase (AC). AC is an important enzyme, which is involved in many signaling pathways. ${ }^{13}$ Its activity is commonly measured by monitoring the

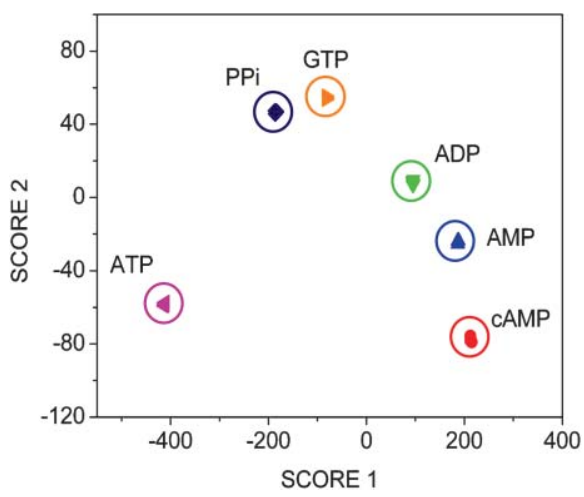

Fig. 4 Two-dimensional LDA score plot for the analysis of five different nucleotides and pyrophosphate. Concentrations: $[$ Mordant Yellow 10] $=$ $[$ Gallocyanine $]=40 \mu \mathrm{M}$, [Evans Blue $]=20 \mu \mathrm{M},[\mathrm{Rh}]=120 \mu \mathrm{M}$, [analyte $]$ $=1.0 \mathrm{mM}, 50 \mathrm{mM}$ phosphate buffer, $\mathrm{pH} 7.4$. 


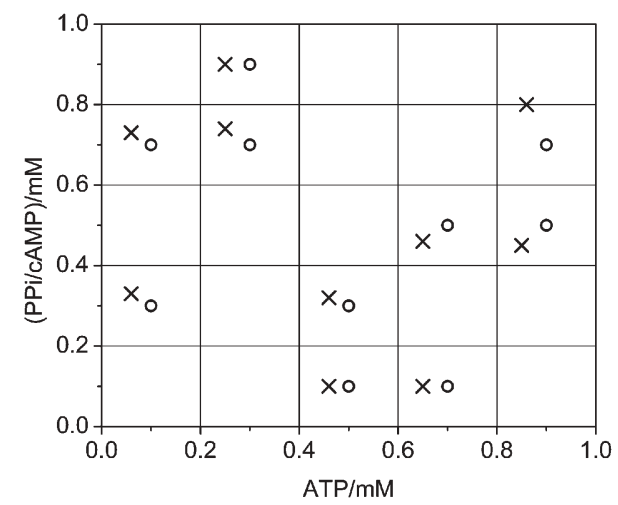

Fig. 5 Actual (circles) and predicted (crosses) concentrations for ternary ATP/cAMP/PPi mixtures $([\mathrm{cAMP}]=[\mathrm{PPi}])$. The intersections of the grid lines represent the 36 points used as the calibration set. The composition of the MIDA is the same as in Fig. 3 and 4.

conversion of $\left[\alpha^{-32} \mathrm{P}\right] \mathrm{ATP}$ to $\left[\alpha-{ }^{32} \mathrm{P}\right] \mathrm{cAMP}$ using ion-exchange chromatography to separate the nucleotides. ${ }^{14}$ To test whether our sensing ensemble could be used to measure the concentrations of ATP and cAMP/PPi simultaneously, we prepared two stock solutions: one with ATP and another one with equal concentrations of cAMP and PPi. By mixing these stock solutions, we then made a calibration set of 36 ternary solutions with ATP and cAMP/PPi concentrations between 0.0 and $1.0 \mathrm{mM}(0.2 \mathrm{mM}$ increments). The calibration set was used to train an artificial neural network employing a standard multi-layer perceptron (MLP) model. ${ }^{15}$ The main component of a MLP is the neuron, a unit which sums the inputs with some weights, and performs a transformation via an activation function, which is responsible for non-linearity. The neuron units are organized in layers and an error back-propagation algorithm is used to train the MLP, i.e. to adapt the weights of the neurons to fit the model to the experimental data. The intensities at five wavelengths $(720,625$, $600,540,440 \mathrm{~nm}$ ) were selected as inputs. Thus, the particular MLP used contained five input neurons, five hidden neurons arranged in one layer, and two output neurons representing the target variables, that is the concentrations of ATP and cAMP/PPi.

To test the predictive power of the trained artificial neural network, we prepared a validation set of ten samples with ATP and cAMP/PPi concentrations between 0.0 and $1.0 \mathrm{mM}$. The actual and predicted concentrations for the validation samples are shown in Fig. 5. Root-mean-square errors were calculated to be $0.045 \mathrm{mM}$ for ATP and $0.042 \mathrm{mM}$ for cAMP/PPi. It is interesting to note that lower concentrations were predicted for ATP for each sample while that was not the case for CAMP/PPi.

In summary, we have shown that a sensing ensemble composed of a Rh complex and three dyes can be used to sense low millimolar concentrations of nucleotides and PPi in buffered aqueous solution. Moreover, the MIDA allows to simultaneously determine the concentrations of ATP and PPi/cAMP with a single $\mathrm{UV}-\mathrm{Vis}$ measurement. The sensor displays a very pronounced selectivity for nucleotides and PPi over phosphate as the analysis can be performed in $50 \mathrm{mM}$ phosphate buffer. From an experimental point of view it is noteworthy that the sensor can be obtained by simply mixing four commercially available compounds. Overall, the results clearly demonstrate the analytical power of MIDAs in combination with pattern-recognition protocols.

The work was supported by the Swiss National Science Foundation (project no. 200021-113944 and no. 200020-111752), by the COST action D31, and by the EPFL.

\section{Notes and references}

\$ Control experiments with complex 1, Evans Blue und ATP confirm that ATP can indeed displace Evans Blue to some extent.

$\S$ The linear discriminant analysis was performed with the help of the following software: SYSTAT (ver. 11.0), Systat Software Inc., Richmond, CA, USA.

- The number of hidden neurons needs to be optimized for a particular problem. Choosing too many hidden neurons may lead to over-fitting. The MLP then loses its ability to generalize the dependency from the data samples. A cross-validation procedure was used to optimize the structure of the MLP and resulted in five hidden neurons.

1 For selected recent examples, see: (a) L. Vial and P. Dumy, J. Am. Chem. Soc., 2007, 129, 4884; (b) H.-W. Rhee, H.-Y. Choi, K. Han and J.-I. Hong, J. Am. Chem. Soc., 2007, 129, 4524; (c) C. Bazzicalupi, A. Bencini, L. Bussotti, E. Berni, S. Biagini, E. Faggi, P. Foggi, C. Giorgi, A. Lapini, A. Marcelli and B. Valtancoli, Chem. Commun., 2007, 1230; (d) C. Li, M. Numata, M. Takeuchi and S. Shinkai, Angew. Chem., Int. Ed., 2005, 44, 6371; (e) S. Yamaguchi, I. Yoshimura, T. Kohira, S. Tamaru and I. Hamachi, J. Am. Chem. Soc., 2005, 127, 11835; (f) C. Yin, F. Gao, F. Huo and P. Yang, Chem. Commun., 2004, 934; (g) D. H. Lee, S. Y. Kim and J.-I. Hong, Angew. Chem., Int. Ed., 2004, 43, 4777; (h) J. Y. Kwon, N. J. Singh, H. N. Kim, S. K. Kim, K. S. Kim and J. Yoon, J. Am. Chem. Soc., 2004, 126, 8892; (i) S. C. McCleskey, M. J. Griffin, S. E. Schneider, J. T. McDevitt and E. V. Anslyn, J. Am. Chem. Soc., 2003, 125, 1114; (j) A. Ojida, S. Park, Y. Mito-oka and I. Hamachi, Tetrahedron Lett., 2002, 43, 6193; $(k)$ S. Mizukami, T. Nagano, Y. Urano, A. Odani and K. Kikuchi, J. Am. Chem. Soc., 2002, 124, 3920.

2 For reviews, see: (a) E. V. Anslyn, J. Org. Chem., 2007, 72, 687; (b) B. T. Nguyen and E. V. Anslyn, Coord. Chem. Rev., 2006, 250, 3118; (c) L. Fabbrizzi, M. Licchelli and A. Taglietti, Dalton Trans., 2003, 3471.

3 (a) A. M. Piątek, Y. J. Bomble, S. L. Wiskur and E. V. Anslyn, J. Am. Chem. Soc., 2004, 126, 6072; (b) L. Fabbrizzi, N. Marcotte, F. Stomeo and A. Taglietti, Angew. Chem., Int. Ed., 2002, 41, 3811.

4 S. L. Wiskur, P. N. Floriano, E. V. Anslyn and J. T. McDevitt, Angew. Chem., Int. Ed., 2003, 42, 2070.

5 S. C. McCleskey, P. N. Floriano, S. L. Wiskur, E. V. Anslyn and J. T. McDevitt, Tetrahedron, 2003, 59, 10089.

6 (a) A. Buryak and K. Severin, J. Comb. Chem., 2006, 8, 540; (b) A. Buryak and K. Severin, Angew. Chem., Int. Ed., 2005, 44, 7935.

7 (a) A. T. Wright and E. V. Anslyn, Chem. Soc. Rev., 2006, 35, 14; (b) K. J. Albert, N. S. Lewis, C. L. Schauer, G. A. Sotzing, S. E. Stizel, T. P. Vaid and D. R. Walt, Chem. Rev., 2000, 100, 2595.

8 A. Buryak and K. Severin, Angew. Chem., Int. Ed., 2004, 43, 4771.

9 A. Buryak and K. Severin, J. Am. Chem. Soc., 2005, 127, 3700.

10 F. Zaubitzer, A. Buryak and K. Severin, Chem.-Eur. J., 2006, 12, 3928.

11 R. H. Fish, Coord. Chem. Rev., 1999, 185-186, 569.

12 P. C. Jurs, G. A. Bakken and H. E. McClelland, Chem. Rev., 2000, 100, 2649.

13 (a) R. K. Sunahara, C. W. Dessauer and A. G. Gilman, Annu. Rev. Pharmocol. Toxicol., 1996, 36, 461; (b) J. L. Bos, Nat. Rev. Mol. Cell Biol., 2003, 4, 733.

14 (a) Y. Salomon, C. Londos and M. Rodbell, Anal. Biochem., 1974, 58, 541; (b) M. D. Smigel, J. Biol. Chem., 1986, 261, 1976; (c) R. K. Sunahara, C. W. Dessauer, R. E. Whisnant, C. Kleuss and A. G. Gilman, J. Biol. Chem., 1997, 272, 22265.

15 S. Haykin, Neural Networks: A comprehensive Foundation, Prentice Hall International, NJ, 1999. 